\title{
Conformal Mappings in Geometric Algebra
}

\author{
Garret Sobczyk
}

I n 1878 William Kingdon Clifford wrote down the rules for his geometric algebra, also known as Clifford algebra. We argue in this paper that in doing so he laid down the groundwork that is profoundly altering the language used by the mathematical community to express geometrical ideas. In the real estate business everyone knows that what is most important is location. We demonstrate here that in the business of mathematics what is most important to the clear and concise expression of geometrical ideas is notation. In the words of Bertrand Russell,

...A good notation has a subtlety and suggestiveness which at times make it seem almost like a live teacher.

Heinrich Hertz expressed much the same thought when he said,

One cannot escape the feeling that these mathematical formulae have an independent existence and an intelligence of their own, that they are wiser than we are, wiser even than their discoverers, that we get more out of them than we originally put into them.

The development of the real and complex number systems represents a hard-won milestone in the robust history of mathematics over many centuries and many different civilizations [5], [29]. Without it mathematics could progress only haltingly, as is evident from the history of mathematics and even the terminology that we use today. Negative numbers were referred to by Rene Descartes (1596-1650) as "fictitious", and "imaginary" numbers were held up to even greater ridicule, though they were first conceived as early as Heron of

Garret Sobczyk is professor of mathematics at Universidad de Las Américas-Puebla. His email address is gar ret_ sobczyk@yahoo.com .

DOI: http://dx.doi.org/10.1090/noti793

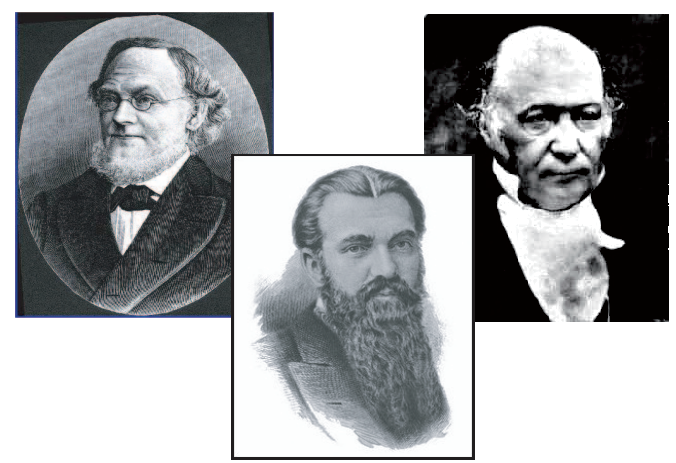

Herman Gunther Grassmann (1809-1877) was a high school teacher. His far-reaching

Ausdehnungslehre, "Theory of extension", laid the groundwork for the development of the exterior or outer product of vectors. William

Rowan Hamilton (1805-1865) was an Irish physicist, astronomer, and mathematician. His invention of the quaternions as the natural generalization of the complex numbers of the plane to three-dimensional space, together with the ideas of Grassmann, set the stage for William Kingdon Clifford's definition of geometric algebra. William Kingdon Clifford (1845-1879) was a professor of mathematics and mechanics at the University College of London. Tragically, he died at the early age of 34 before he could explore his profound ideas.

Alexandria, the illustrious inventor of the windmill and steam engine during the first century $\mathrm{AD}$ [18]. What most mathematicians fail to see even today is that geometric algebra represents the grand culmination of that process with the completion of the real number system to include the concept of direction. Geometric algebra combines the two silver currents of mathematics, geometry and algebra, into a single coherent language. As David Hestenes has eloquently stated, 


\section{Algebra without geometry is blind, geometry without algebra is dumb.}

David Hestenes, a newly hired assistant professor of physics, introduced me to geometric algebra at Arizona State University in 1966. I immediately became captivated by the idea that geometric algebra is the natural completion of the real number system to include the concept of direction. If this idea is correct, it should be possible to formulate all the ideas of multilinear linear algebra, tensor analysis, and differential geometry. I considered it to be a major breakthrough when I succeeded in proving the fundamental Cayley-Hamilton theorem within the framework of geometric algebra. The next task was mastering differential geometry. Five years later, with Hestenes as my thesis director, I completed my doctoral dissertation, "Mappings of Surfaces in Euclidean Space Using Geometric Algebra" [26].

Much work of Hestenes and other theoretical physicists has centered around understanding the geometric meaning of the appearance of imaginary numbers in quantum mechanics. Ed Witten, in his Josiah Willard Gibbs Lecture "Magic, Mystery, and Matrix" says, "Physics without strings is roughly analogous to mathematics without complex numbers" [31]. In a personal communication with the author, Witten points out that "Pierre Ramond, in 1970, generalized the Clifford algebra from field theory (where Dirac had used it) to string theory." Dale Husemöller in his book Fibre Bundles includes a chapter on Clifford algebras, recognizing their importance in "topological applications and for giving a concrete description of the group Spin(n)" [13]. Other mathematicians have developed an extensive new field which has come to be known as "Clifford analysis" [3], [7] and "spin geometry" [2].

During the academic year 1972-73 I continued work at Arizona State University with David on differential geometry as a visiting assistant professor. The following year, at the height of the academic crunch, I was on my own-a young unemployed mathematician, but with a mission: To convince the world of the importance of geometric algebra. I travelled to Europe and European universities giving talks when and where I was graciously invited. During the year 1973-74, Professor Roman Duda of the Polish Academy of Sciences offered me a postgraduate research position, which I gratefully accepted.

My collaboration with David culminated a number of years later with the publication of our book Clifford Algebra to Geometric Calculus: A Unified Language for Mathematics and Physics [11]. Over the years I have been searching for the best way to handle ideas in linear algebra and differential geometry, particularly with regard to introducing students to geometric algebra at the undergraduate level. To this end, I have been working to complete a new manuscript New Foundations in Mathematics: The Geometric Concept of Numbers which has been accepted for publication by Birkhäuser [24].

Distilling geometric algebra down to its core, it is the extension of the real number system to include new anticommuting square roots of \pm 1 , which represent mutually orthogonal unit vectors in successively higher dimensions. Whereas everybody is familiar with the idea of the imaginary number $i=\sqrt{-1}$, the idea of a new square root $u=\sqrt{+1} \notin \mathbb{R}$ has taken longer to be assimilated into the main body of mathematics. However, in [22] it is shown that the hyperbolic numbers, which have much in common with their more famous brothers the complex numbers, also lead to an efficacious solution of the cubic equation.

The time has come for geometric algebra to become universally recognized for what it is: the completion of the real number system to include the concept of direction. Let twenty-first century mathematics be a century of consolidation and streamlining so that it can bloom with all the renewed vigor that it will need to prosper in the future [10].

\section{Fundamental Concepts}

Let $\mathbb{R}^{p, q}$ be the pseudoeuclidean space of the indefinite nondegenerate real quadratic form $q(\mathbf{x})$ with signature $\{p, q\}$, where $\mathbf{x} \in \mathbb{R}^{p, q}$. With this quadratic form, we define the inner product $\mathbf{x} \cdot \mathbf{y}$ for $\mathbf{x}, \mathbf{y} \in \mathbb{R}^{p, q}$ to be

$$
\mathbf{x} \cdot \mathbf{y}=\frac{1}{2}[q(\mathbf{x}+\mathbf{y})-q(\mathbf{x})-q(\mathbf{y})] .
$$

Let $(\mathbf{e})_{(n)}=\left(\mathbf{e}_{1}, \mathbf{e}_{2}, \ldots, \mathbf{e}_{p+q}\right)$ denote the row of standard orthonormal anticommuting basis vectors of $\mathbb{R}^{p, q}$, where $\mathbf{e}_{j}^{2}=q\left(\mathbf{e}_{j}\right)$ for $j=1, \ldots, n$, i.e.,

$$
\mathbf{e}_{1}^{2}=\cdots=\mathbf{e}_{p}^{2}=1 \text { and } \mathbf{e}_{p+1}^{2}=\cdots=\mathbf{e}_{p+q}^{2}=-1
$$

and $n=p+q$. Taking the transpose of the row of basis vectors $(\mathbf{e})_{(n)}$ gives the column $(\mathbf{e})_{(n)}^{T}$ of these same basis vectors. The metric tensor $[\mathrm{g}]$ of $\mathbb{R}^{p, q}$ is defined to be the Gramian matrix

$$
[g]:=(\mathbf{e})_{(n)}^{T} \cdot(\mathbf{e})_{(n)},
$$

which is a diagonal matrix with the first $p$ entries +1 and the remaining entries -1 . The standard reciprocal basis to $(\mathbf{e})_{(n)}$ is then defined, with raised indices, by

$$
(\mathbf{e})^{(n)}=[g](\mathbf{e})_{(n)}^{T} .
$$

In terms of the standard basis $(\mathbf{e})_{(n)}$, and its reciprocal basis $(\mathbf{e})^{(n)}$, a vector $\mathbf{x} \in \mathbb{R}^{p, q}$ is efficiently expressed by

$$
\mathbf{X}=(\mathbf{e})_{(n)}(x)^{(n)}=(x)_{(n)}(\mathbf{e})^{(n)} .
$$

Both the column vector of components $(x)^{(n)}$ and the row vector of components of $\mathbf{x}$ are, of course, 


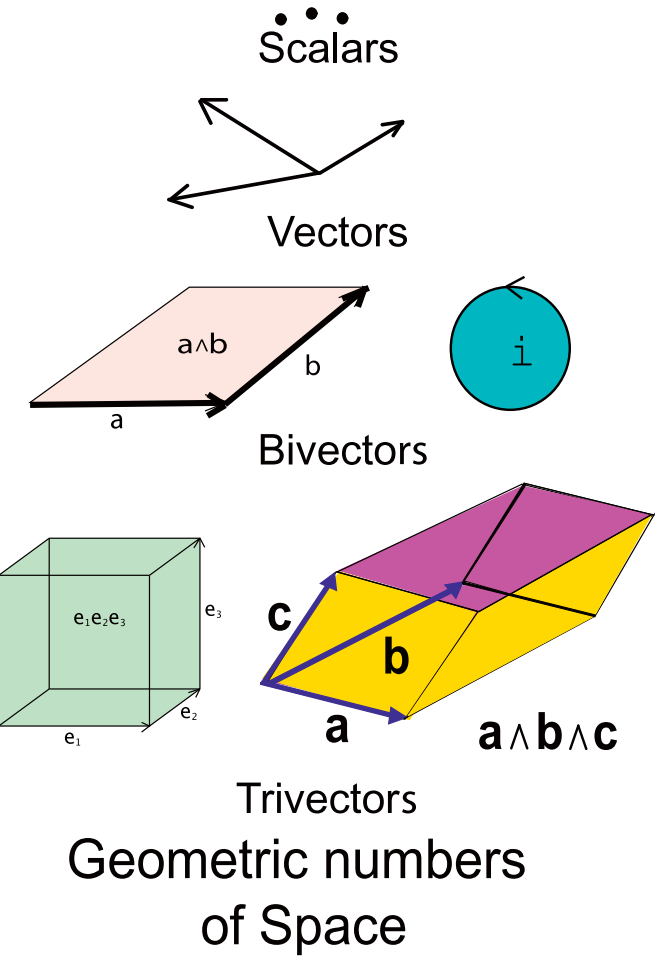

Figure 1.

real numbers. If $\mathbf{y}=(\mathbf{e})_{(n)}(y)^{(n)}$ is a second such vector, then the inner product between them is

$$
\begin{aligned}
& \mathbf{x} \cdot \mathbf{y}=(x)_{(n)}(\mathbf{e})^{(n)} \cdot(\mathbf{e})_{(n)}(y)^{(n)} \\
& =\sum_{i=1}^{n} x_{i} y^{i}=\sum_{i=1}^{p} x_{i} y_{i}-\sum_{j=p+1}^{n} x_{j} y_{j},
\end{aligned}
$$

where $n=p+q$.

Let $(\mathbf{a})_{(n)}$ be a second basis of $\mathbb{R}^{p, q}$. Then

$$
(\mathbf{a})_{(n)}=(\mathbf{e})_{(n)} A \text { and }(\mathbf{a})_{(n)}^{T}=A^{T}(\mathbf{e})_{(n)}^{T} \text {, }
$$

where $A$ is the matrix of transition from the standard basis $(\mathbf{e})_{(n)}$ of $\mathbb{R}^{p, q}$ to the basis $(\mathbf{a})_{(n)}$ of $\mathbb{R}^{p, q}$. The Gramian matrix of inner products of the basis vectors $(\mathbf{a})_{(n)}$ is then given by

$$
(\mathbf{a})_{(n)}^{T} \cdot(\mathbf{a})_{(n)}=A^{T}(\mathbf{e})_{(n)}^{T} \cdot(\mathbf{e})_{(n)} A=A^{T}[g] A
$$

where $[g]$ is the metric tensor of $\mathbb{R}^{p, q}$ defined in (1).

The real associative geometric algebra $\mathbb{G}_{p, q}=$ $\mathbb{G}_{p, q}\left(\mathbb{R}^{p, q}\right)$ is generated by taking sums of geometric products of the vectors in $\mathbb{R}^{p, q}$, subjected to the rule that $\mathbf{x}^{2}=q(\mathbf{x})=\mathbf{x} \cdot \mathbf{x}$ for all vectors $\mathbf{x} \in \mathbb{R}^{p, q}$. The dimension of $\mathbb{G}_{p, q}$ as a real linear space is $2^{n}$, where $n=p+q$, with the standard orthonormal basis of geometric numbers

$$
\mathbb{G}_{p, q}=\operatorname{span}\left\{\mathbf{e}_{\lambda_{k}}\right\}_{k=0}^{n},
$$

where the $\left(\begin{array}{l}n \\ k\end{array}\right) k$-vector basis elements of the form $\mathbf{e}_{\lambda_{k}}$ are defined by

$$
\mathbf{e}_{\lambda_{k}}=\mathbf{e}_{i_{1} \ldots i_{k}}=\mathbf{e}_{i_{1}} \cdots \mathbf{e}_{i_{k}}
$$

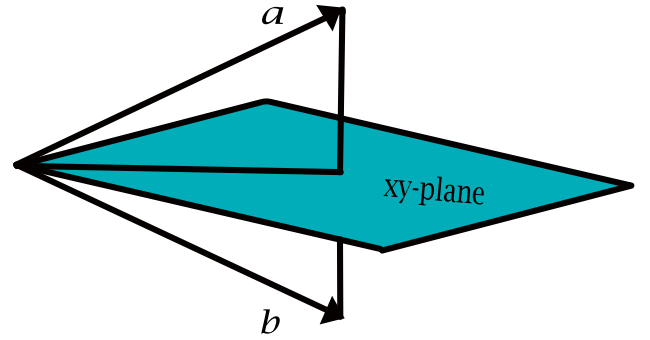

Figure 2 . The vector $a$ is reflected in the $x y$-plane to give the vector $b=e_{12} \mathbf{a e}_{12}$, where

$\mathbf{e}_{1}$ and $\mathbf{e}_{2}$ are unit vectors along the $x$ and $y$ axis, respectively, and $e_{12}$ is the unit bivector of the $x y$-plane.

for each $\lambda_{k}=i_{1} \ldots i_{k}$ where $1 \leq i_{1}<\cdots<i_{k} \leq n$. When $k=0, \lambda_{0}=0$ and $\mathbf{e}_{0}=1$. For example, the $2^{3}$-dimensional geometric algebra $\mathbb{G}_{3}$ of $\mathbb{R}^{3}$ has the standard basis

$$
\begin{aligned}
\mathbb{G}_{3} & =\operatorname{span}\left\{\mathbf{e}_{\lambda_{k}}\right\}_{k=0}^{3} \\
& =\operatorname{span}\left\{1, \mathbf{e}_{1}, \mathbf{e}_{2}, \mathbf{e}_{3}, \mathbf{e}_{12}, \mathbf{e}_{13}, \mathbf{e}_{23}, \mathbf{e}_{123}\right\} .
\end{aligned}
$$

The geometric numbers of space are pictured in Figure 1.

The great advantage of geometric algebra is that deep geometrical relationships can be expressed directly in terms of the multivectors of the algebra without having to constantly refer to a basis; see Figures 2 and 3. On the other hand, the language gives geometric legs to the powerful matrix formalism that has developed over the last one hundred fifty years. As a real associative algebra, each geometric algebra under geometric multiplication is isomorphic to a corresponding algebra or subalgebra of real matrices, and, indeed, we have advocated elsewhere the need for a uniform approach to both of these structures [23], [27]. Matrices are invaluable for systematizing calculations, but geometric algebra provides deep geometrical insight and powerful algebraic tools for the efficient expression of geometrical properties. Clifford algebras and their relationships to matrix algebra and the classical groups have been thoroughly studied in [15], [21] and elsewhere.

Whereas it is impossible to summarize all of the identities in geometric algebra that we need here, there are many good references that are available, for example, [1], [6], [9], [11], [15]. The most basic identity is the breaking down of the geometric product of two vectors $\mathbf{x}, \mathbf{y} \in \mathbb{R}^{p, q}$ into the symmetric and skewsymmetic parts, giving

(3) $\mathbf{x y}=\frac{1}{2}(\mathbf{x y}+\mathbf{y} \mathbf{x})+\frac{1}{2}(\mathbf{x y}-\mathbf{y} \mathbf{x})=\mathbf{x} \cdot \mathbf{y}+\mathbf{x} \wedge \mathbf{y}$,

where the inner product $\mathbf{x} \cdot \mathbf{y}$ is the symmetric part and the outer product $\mathbf{x} \wedge \mathbf{y}$ is the skewsymmetric part. For whatever the reason, historical or otherwise, the importance of this identity for mathematics has yet to be fully appreciated. The outer 


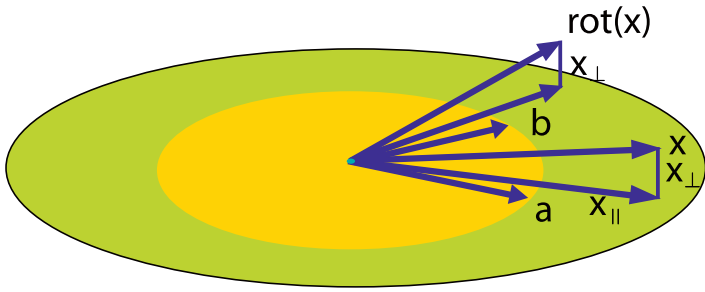

Figure 3. The vector $\mathbf{x}=\mathbf{x}_{\|}+\mathbf{x}_{\perp}$ is rotated in the plane of the bivector $\mathbf{a} \wedge \mathbf{b}$ into $\operatorname{rot}(\mathbf{x})$, leaving the perpendicular component unchanged. When $|\mathbf{a}|=|\mathbf{b}|=\mathbf{1}$, $\operatorname{rot}(\mathbf{x})=e^{-i \frac{\theta}{2}} \mathbf{x} e^{i \frac{\theta}{2}}$, where $e^{i \frac{\theta}{2}}=\frac{\mathbf{a}(\mathbf{a}+\mathbf{b})}{|\mathbf{a}+\mathbf{b}|}$, $e^{-i \frac{\theta}{2}}=\frac{(a+b) a}{|a+b|}$ and $\theta$ is the angle between $\mathbf{a}$ and b. If $\mathbf{a}$ and $b$ lie in the $x y$-plane, then $i=\mathbf{e}_{12}$.

product $\mathbf{x} \wedge \mathbf{y}$ has the geometric interpretation of a bivector or a directed plane segment.

Other, more general but similar, identities are quickly developed. In general, the geometric product between a vector $\mathbf{a}$ and a $k$-vector $\mathbf{B}_{k}$, for $k \geq 1$, can always be decomposed into the sum of an inner product (contraction) and an outer product (extension),

$$
\mathbf{a B}_{k}=\mathbf{a} \cdot \mathbf{B}_{k}+\mathbf{a} \wedge \mathbf{B}_{k}
$$

The inner product $\mathbf{a} \cdot \mathbf{B}_{k}$ is a $(k-1)$-vector, defined by

$$
\mathbf{a} \cdot \mathbf{B}_{k}:=\frac{1}{2}\left(\mathbf{a B}_{k}+(-1)^{k+1} \mathbf{B}_{k} \mathbf{a}\right)=\left\langle\mathbf{a} \mathbf{B}_{k}\right\rangle_{k-1},
$$

and the outer product $\mathbf{a} \wedge \mathbf{B}_{k}$ is $\mathbf{a}(k+1)$-vector, defined by

$$
\mathbf{a} \wedge \mathbf{B}_{k}:=\frac{1}{2}\left(\mathbf{a B}_{k}-(-1)^{k+1} \mathbf{B}_{k} \mathbf{a}\right)=\left\langle\mathbf{a B}_{k}\right\rangle_{k+1} .
$$

More generally, if $\mathbf{A}_{r}$ and $\mathbf{B}_{s}$ are $r$ - and $s$-vectors of $\mathbb{G}_{p, q}$, where $r>0, s>0$, we define

$$
\mathbf{A}_{r} \cdot \mathbf{B}_{s}=\left\langle\mathbf{A}_{r} \mathbf{B}_{s}\right\rangle_{|r-s|}
$$

and

$$
\mathbf{A}_{r} \wedge \mathbf{B}_{s}=\left\langle\mathbf{A}_{r} \mathbf{B}_{s}\right\rangle_{r+s}
$$

In the exceptional cases when $r=0$ or $s=0$, we define $\mathbf{A}_{r} \cdot \mathbf{B}_{s}=0$ and $\mathbf{A}_{r} \wedge \mathbf{B}_{s}=\mathbf{A}_{r} \mathbf{B}_{s}$.

For the vectors $\mathbf{a}$ and $\mathbf{b}$ and the $k$-vector $\mathbf{C}_{k}$, where $k \geq 1$, we now show that

$$
\mathbf{a} \cdot\left(\mathbf{b} \wedge \mathbf{C}_{k}\right)=(\mathbf{a} \cdot \mathbf{b}) \mathbf{C}_{k}-\mathbf{b} \wedge\left(\mathbf{a} \cdot \mathbf{C}_{k}\right) .
$$

Using the above definitions, we decompose the left side of this equation, getting $\mathbf{a} \cdot\left(\mathbf{b} \wedge \mathbf{C}_{k}\right)=$

$$
\frac{1}{4}\left[\mathbf{a b C}_{k}+(-1)^{k} \mathbf{a C} \mathbf{C}_{k} \mathbf{b}+(-1)^{k} \mathbf{b} \mathbf{C}_{k} \mathbf{a}+\mathbf{C}_{k} \mathbf{b a}\right] .
$$

Decomposing the right-hand side of the equation gives

$$
\begin{gathered}
(\mathbf{a} \cdot \mathbf{b}) \mathbf{C}_{k}-\mathbf{b} \wedge\left(\mathbf{a} \cdot \mathbf{C}_{k}\right)= \\
\frac{1}{2}\left[(\mathbf{a} \cdot \mathbf{b}) \mathbf{C}_{k}+\mathbf{C}_{k}(\mathbf{a} \cdot \mathbf{b})-\mathbf{b}\left(\mathbf{a} \cdot \mathbf{C}_{k}\right)+(-1)^{k}\left(\mathbf{a} \cdot \mathbf{C}_{k}\right) \mathbf{b}\right] \\
=\frac{1}{4}\left[(\mathbf{a b}+\mathbf{b a}) \mathbf{C}_{k}+\mathbf{C}_{k}(\mathbf{a b}+\mathbf{b a})-\mathbf{b a C}_{k}\right. \\
\left.+(-1)^{k} \mathbf{b} \mathbf{C}_{k} \mathbf{a}+(-1)^{k} \mathbf{a} \mathbf{C}_{k} \mathbf{b}-\mathbf{C}_{k} \mathbf{a b}\right] .
\end{gathered}
$$

After cancellations, we see that the left and right sides of the equations are identical, so the identity (5) is proved.

One very useful identity which follows from (5) is

$$
\begin{aligned}
\mathbf{a} \cdot\left(\mathbf{A}_{r} \wedge \mathbf{B}_{s}\right) & =\left(\mathbf{a} \cdot \mathbf{A}_{r}\right) \wedge B_{S}+(-1)^{r} \mathbf{A}_{r} \wedge\left(\mathbf{a} \cdot \mathbf{B}_{s}\right) \\
= & (-1)^{r+s+1}\left(\mathbf{A}_{r} \wedge \mathbf{B}_{s}\right) \cdot \mathbf{a} .
\end{aligned}
$$

It gives the distributive law for the inner product of a vector over the outer product of an $r$-and an $s$-vector.

The outer product of $k$ vectors $\mathbf{a}_{i} \in \mathbb{R}^{p, q}$ is defined by

$$
\mathbf{a}_{(k)}:=\wedge(\mathbf{a})_{(k)}=\mathbf{a}_{1} \wedge \cdots \wedge \mathbf{a}_{k} .
$$

The outer product can be directly expressed as the completely antisymmetric geometric product of those vectors. We have

$$
\mathbf{a}_{1} \wedge \cdots \wedge \mathbf{a}_{k}=\sum_{\pi \in \Pi} \operatorname{sgn}(\pi) \frac{1}{k !} \mathbf{a}_{\pi(1)} \cdots \mathbf{a}_{\pi(k)},
$$

where $\pi$ is a permutation on the indices $(1,2$, $\ldots, k)$, and $\operatorname{sgn}(\pi)= \pm 1$ according to whether $\pi$ is an even or odd permutation, respectively.

As an application of our notation and our change of basis formula (2), for $(\mathbf{a})_{(n)}=(\mathbf{e})_{(n)} A$, we have

$$
\mathbf{a}_{(n)}=\mathbf{a}_{1} \wedge \cdots \wedge \mathbf{a}_{n}=\wedge(\mathbf{a})_{(n)}=\mathbf{e}_{(n)} \operatorname{det} A .
$$

By the reverse $A^{\dagger}$ of the geometric number $A \in \mathbb{C}_{p, q}$, we mean the geometric number obtained by reversing the order of the vectors in all of the geometric products that make up $A[15$, p. 29]. For example, if $A=\mathbf{a}_{1}+\mathbf{a}_{1} \mathbf{a}_{2} \mathbf{a}_{3}$, then $A^{\dagger}=\mathbf{a}_{1}+\mathbf{a}_{3} \mathbf{a}_{2} \mathbf{a}_{1}$. If $A, B \in \mathbb{G}_{p, q}$, then it immediately follows that

$$
(A+B)^{\dagger}=A^{\dagger}+B^{\dagger} \text { and }(A B)^{\dagger}=B^{\dagger} A^{\dagger} .
$$

By the magnitude $|A|$ of a geometric number $A \in \mathbb{G}_{p, q}$, we mean

$$
|A|=\sqrt{\left|\left\langle A A^{\dagger}\right\rangle_{0}\right|} \geq 0
$$

In the case of any geometric number in the geometric algebra $\mathbb{C}_{n}$ of the Euclidean space $\mathbb{R}^{n}$, the absolute value inside the square root can be removed.

There are many very useful relationships that can be derived and that can be found in the references previously given. The above identities indicate the richness of the geometric algebra $\mathbb{G}_{p, q}$ as a tool for studying properties of the pseudoeuclidean space $\mathbb{R}^{p, q}$. Figure 4 illustrates one application of geometric algebra to physics [24], whereas Figure 5 sets the stage for directed 


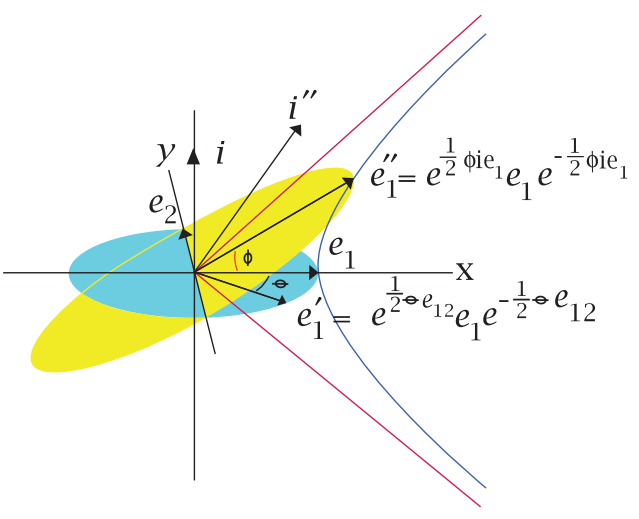

Figure 4. The vector $e_{1}$ is rotated in the $x y$-plane of $\mathbf{e}_{12}$ into the vector $\mathbf{e}_{1}^{\prime}$. The vector $\mathbf{e}_{1}$ is boosted into the relative vector $\mathbf{e}_{1}^{\prime \prime}$ of the relative plane of the relative bivector $\mathbf{e}_{12}^{\prime \prime}=\mathbf{e}_{1}^{\prime \prime} \mathbf{e}_{2}$. The relative plane of $\mathbf{e}_{12}^{\prime \prime}$ has the relative velocity of $\frac{\mathbf{v}}{c}=i \mathrm{e}_{1} \tanh \phi$ with respect to the plane of $\mathbf{e}_{12}$, where $c$ is the speed of light.

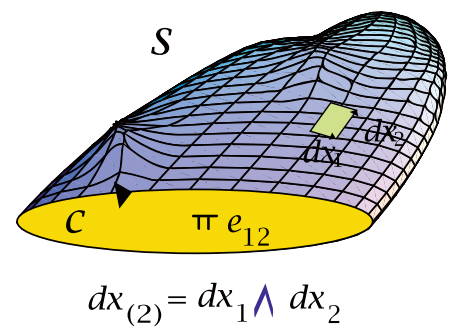

Figure 5. When the element of directed area $d \mathbf{x}_{(2)}=d \mathbf{x}_{1} \wedge d \mathbf{x}_{2}$ is integrated over the smooth oriented surface $S$ bounded by the oriented unit circle $C$ in the $x y$-plane, we get $\int_{S} d \mathbf{x}_{(2)}=\pi \mathbf{e}_{12}$. This is the basis for directed integration theory and Clifford analysis.

integration theory and Clifford analysis, [3] and [11].

\section{Differentiation on Vector Manifolds}

Let $U \subset \mathbb{R}^{p, q}$ be an open set in the relative topology of $\mathbb{R}^{p, q}$ containing the point $\mathbf{x}$, and let $n=p+q$. A geometric-valued function $F: U \rightarrow \mathbb{G}_{p, q}$ is said to be differentiable at $\mathbf{x} \in U$ if and only if there exists a linear map $F_{\mathbf{a}}: \mathbb{R}^{p, q} \rightarrow \mathbb{G}_{p, q}$ such that

$$
\lim _{\mathbf{v} \rightarrow 0, \mathbf{v}^{2} \neq 0} \frac{\left|F(\mathbf{x}+\mathbf{v})-F(\mathbf{x})-F_{\mathbf{v}}(\mathbf{x})\right|}{|\mathbf{v}|}=0 .
$$

The map $F_{\mathbf{a}}(\mathbf{x})$ is linear in the tangent vector $\mathbf{a} \in \mathbb{R}^{p, q}$ and uniquely determined by the local properties of $F$ at the point $\mathbf{x} \in \mathbb{R}^{p, q}$; see [28, p. 16]. For any $\mathbf{a} \in \mathbb{R}^{p, q}$, we write

$$
F_{\mathbf{a}}(\mathbf{x})=\mathbf{a} \cdot \partial_{\mathbf{x}} F(\mathbf{x})
$$

to represent $F_{\mathbf{a}}(\mathbf{x})$ as the more familiar directional derivative of the geometric-valued function $F(\mathbf{x})$ in the direction of the vector $\mathbf{a}$ at the point $\mathbf{x}$.
If $\mathbf{a}=(\mathbf{e})_{(n)}(a)^{(n)} \in \mathbb{R}^{p, q}$ and $\mathbf{x}=(\mathbf{e})_{(n)}(x)^{(n)} \in$ $U$, then

$$
F_{\mathbf{a}}(\mathbf{x})=\sum_{i=1}^{n} a^{i} \frac{\partial F}{\partial x^{i}} .
$$

In differential geometry books, the directional derivative $F_{\mathrm{a}}$ is sometimes referred to as a standard connection on $\mathbb{R}^{p, q}[12$, p. 18].

A differentiable geometric-valued function $F(\mathbf{x})$ has a vector derivative $\partial F$, defined by

$$
\partial F=\partial_{\mathbf{x}} F(\mathbf{x})=\partial_{\mathbf{v}} F_{\mathbf{v}}(\mathbf{x}) .
$$

The second equality on the right expresses the vector derivative in terms of the tangential vector derivative $\partial_{\mathbf{v}}$ of the directional derivative $F_{\mathbf{v}}(\mathbf{x})$ of $F(\mathbf{x})$. In terms of the standard basis of $\mathbb{R}^{p, q}$,

$$
\partial F=\sum_{i=1}^{n} \mathbf{e}_{i} \frac{\partial F}{\partial x^{i}} \text {. }
$$

Just as in (4), the vector derivative $\partial F$ of a $k$ vector-valued function $F$ can be decomposed into the sum of a $(k-1)$-vector part $\partial \cdot F$, called the divergence, and a $(k+1)$-vector part $\partial \wedge F$, called the curl. We have

$$
\partial F=\partial \cdot F+\partial \wedge F
$$

The important condition of integrability has an easy expression in terms of the vector derivative on $\mathbb{R}^{p, q}$,

$$
\partial_{\mathrm{x}} \wedge \partial_{\mathrm{x}}=0,
$$

and is simply an expression of the fact that partial derivatives commute and that the standard basis vectors $\mathbf{e}_{i}$ are constant. Let $\mathbf{a}, \mathbf{b} \in \mathbb{R}^{p, q}$. In terms of directional derivatives, the integrability condition takes the equivalent form

(10) $(\mathbf{a} \wedge \mathbf{b}) \cdot\left(\partial_{\mathbf{x}} \wedge \partial_{\mathbf{x}}\right)=[\mathbf{b} \cdot \partial, \mathbf{a} \cdot \partial]-[\mathbf{b}, \mathbf{a}] \cdot \partial=0$,

where $[\mathbf{b} \cdot \partial, \mathbf{a} \cdot \partial]:=\mathbf{b} \cdot \partial \mathbf{a} \cdot \partial-\mathbf{a} \cdot \partial \mathbf{b} \cdot \partial$, and $[\mathbf{b}, \mathbf{a}]=\mathbf{b} \cdot \partial \mathbf{a}-\mathbf{a} \cdot \partial \mathbf{b}$ is the Lie bracket of the vector fields $\mathbf{a}, \mathbf{b}$. Of course, if $\mathbf{a}$ and $\mathbf{b}$ are constant vectors and are not a function of the point $\mathbf{x} \in \mathbb{R}^{p, q}$, then the Lie bracket $[\mathbf{a}, \mathbf{b}]=0$. In this case, the integrability condition (10) reduces to the condition that the directional derivatives defined by the vectors $\mathbf{a}$ and $\mathbf{b}$ commute.

The definition of the directional and vector derivatives are independent of the signature $p, q$ of the metric involved. A few basic formulas which are valid in any signature and can be easily established, are

$$
\mathbf{a} \cdot \partial_{\mathrm{x}} \mathbf{x}=\mathbf{a}=\partial_{\mathrm{x}} \mathbf{x} \cdot \mathbf{a} \Longrightarrow \partial_{\mathrm{x}} \mathbf{x}=n .
$$

For all integers $r$,

$$
\mathbf{a} \cdot \partial_{\mathbf{x}} \mathbf{x}^{2 r}=2 r(\mathbf{a} \cdot \mathbf{x}) \mathbf{x}^{2 r-2} \Longrightarrow \partial_{\mathbf{x}} \mathbf{x}^{2 r}=2 r \mathbf{x}^{2 n-1}
$$

and

$$
\begin{gathered}
\mathbf{a} \cdot \partial_{\mathbf{X}} \mathbf{x}^{2 r+1}=\left(2 r(\mathbf{a} \cdot \mathbf{x}) \mathbf{x}+\mathbf{a} \mathbf{x}^{2}\right) \mathbf{x}^{2 r-2} \\
\Longrightarrow \partial_{\mathbf{x}} \mathbf{x}^{2 r+1}=(2 r+n) \mathbf{x}^{2 r} \\
\partial_{\mathbf{x}} \mathbf{a x}=\partial_{\mathbf{x}}(\mathbf{x} \cdot \mathbf{a}-\mathbf{x} \wedge \mathbf{a})=-(n-2) \mathbf{a} .
\end{gathered}
$$


In light of the Whitney-Nash embedding theorems [4], [19], [20], [32], [30], little or nothing is lost by assuming that a general manifold is embedded in $\mathbb{R}^{p, q}$. We call such manifolds vector manifolds. By making such an assumption, all the tools of the geometric algebra $\mathbb{G}_{p, q}$ of $\mathbb{R}^{p, q}$ immediately become available for the study of the given vector manifold.

Let $\mathcal{M}$ be a regular $k$-dimensional manifold in $\mathbb{R}^{p, q}$, with an associated atlas of charts specified with respect to the inherited topology from $\mathbb{R}^{p, q}$. Let $U$ be an open set in $\mathcal{M}$ containing the point $\mathbf{x} \in \mathcal{M}$. In some chart whose range contains the open set $U$, the point $\mathbf{x}=\mathbf{x}\left(s^{1}, \ldots, s^{k}\right)$ is defined by the local coordinates $s^{i} \in \mathbb{R}$ for $i=1, \ldots, k$. The beauty of the definitions (7) and (8) is that they can be easily extended to define the Koszul connection and vector derivative on the embedded manifold $\mathcal{M}$ in $\mathbb{R}^{p, q}$; we simply require in the limit that $\mathbf{v} \rightarrow 0$ in such a way that $\mathbf{x}+\mathbf{v} \in U \subset \mathcal{M}$. See [14, pp. 15253] and, in particular, Professor Lee's “confession". For this reason, we use the same symbols for the directional derivatives $F_{\mathbf{a}}(\mathbf{x})$ and the vector derivative $\partial_{\mathbf{x}} F(\mathbf{x})$ of the geometric-valued function $F(\mathbf{x})$ on $\mathcal{M}$.

The standard basis of the tangent space $\mathcal{T}_{\mathbf{x}}^{1}$ of $\mathcal{M}$, with respect to the chart containing $U$, is $(\mathbf{x})_{(k)}=\left(\mathbf{x}_{1}, \mathbf{x}_{2}, \ldots, \mathbf{x}_{k}\right)$, where $\mathbf{x}_{i}=\frac{\partial \mathbf{x}}{\partial s^{i}}$ and the geometric algebra $\mathcal{T}_{\mathrm{x}}$ at the point $\mathbf{x} \in U$ is the subgeometric algebra of $\mathbb{T}_{p, q}$ generated by taking sums of geometric products of the tangent vectors $\mathbf{x}_{i}$. More care must be taken in writing down the integrability condition for the Koszul derivative $\mathbf{a} \cdot \partial_{\mathbf{x}}$ for $\mathbf{a} \in \mathcal{T}_{\mathbf{x}}$. For an embedded curved manifold $\mathcal{M}$, (9) no longer remains valid, but the alternative condition (10) does remain valid.

\section{Projection and Curvature}

Two of the most important objects on a vector manifold are the unit pseudoscalar field, defined by

$$
I=I_{\mathbf{x}}=\frac{\mathbf{x}_{1} \wedge \cdots \wedge \mathbf{x}_{k}}{\left|\mathbf{x}_{1} \wedge \cdots \wedge \mathbf{x}_{k}\right|},
$$

and the projection $P=P_{\mathrm{x}}: \mathbb{G}_{p, q} \rightarrow \mathcal{T}_{\mathrm{x}}$ onto the tangent algebra $\mathcal{T}_{\mathbf{x}}$ at the point $\mathbf{x} \in \mathcal{M}$, defined by

$$
P\left(A_{r}\right)=\left(A_{r} \cdot I\right) I^{\dagger},
$$

where $A_{r} \in \mathbb{G}_{p, q}^{r}$ is any $r$-vector, for $1 \leq r \leq n$. When $r=0$, the projection acts as the identity, $P_{\mathbf{x}}(\alpha)=\alpha$ for all $\alpha \in \mathbb{R}$. A smooth vector manifold $\mathcal{M}$ is said to be orientable if $I_{\mathrm{x}}$ is single valued at all points $\mathbf{x} \in \mathcal{M}$.

Note from (4) that the projection operator can be defined entirely in terms of the geometric product without the use of the inner or outer products. This guarantees that all the rules for the differentiation of geometric-valued functions are the same as for the differentiation of scalar-valued functions. However, the noncommutativity of the geometric product must always be respected. This means that the projection $P_{\mathrm{x}}$ can be differentiated in the same way as other geometric-valued functions. In particular, we define

$$
P_{\mathbf{a}}\left(A_{r}\right):=\mathbf{a} \cdot \partial_{\mathbf{x}} P_{\mathbf{x}}\left(A_{r}\right)-P_{\mathbf{x}}\left(\mathbf{a} \cdot \partial_{\mathbf{x}} A_{r}\right)
$$

and

$P_{\mathbf{a}, \mathbf{b}}\left(A_{r}\right):=\mathbf{b} \cdot \partial_{\mathbf{x}} P_{\mathbf{a}}\left(A_{r}\right)-P_{\mathbf{b} \cdot \partial_{\mathbf{a}}}\left(A_{r}\right)-P_{\mathbf{x}}\left(\mathbf{b} \cdot \partial_{\mathbf{x}} A_{r}\right)$, thus ensuring the linearity of $P_{\mathbf{a}, \mathbf{b}}\left(A_{r}\right)$ in each of its arguments $\mathbf{a}, \mathbf{b}$ and $A_{r}$. Note that the integrability condition (10) tells us that

$$
P_{\mathbf{a}, \mathbf{b}}-P_{\mathbf{b}, \mathbf{a}}=(\mathbf{a} \wedge \mathbf{b}) \cdot(\partial \wedge \partial) P_{\mathbf{x}}=0 .
$$

The tangent pseudoscalar $I_{\mathrm{x}}$ and the projection $P_{\mathrm{x}}$ defined in terms of it can be used to completely characterize both the intrinsic and extrinsic curvature of the vector manifold $\mathcal{M}$ as it is situated in the flat pseudoeuclidean space $\mathbb{R}^{p, q}$. The shape operator $S: \mathbb{G}_{p, q} \rightarrow \mathbb{G}_{p, q}$ at the point $\mathbf{x} \in U \subset \mathcal{M}$ is defined by

$$
S(A):=\partial_{\mathbf{x}} P_{\mathbf{x}}(A)=\partial_{\mathbf{v}} P_{\mathbf{v}}(A),
$$

for each $A \in \mathbb{G}_{p, q}$. The shape operator is a generalization of the classical Weingarten map, which is defined for hypersurfaces in $\mathbb{R}^{p, q}[14, \mathrm{p} .155]$.

The shape operator $S$ completely determines the classical Riemann curvature tensor. By the Riemann curvature bivector we mean

$$
R(\mathbf{a} \wedge \mathbf{b})=P_{\mathbf{a}}(S(\mathbf{b}))=\partial_{\mathbf{v}} \wedge P_{\mathbf{a}} P_{\mathbf{b}}(\mathbf{v}),
$$

where $\mathbf{a}, \mathbf{b}$ are vectors in the tangent space $\mathcal{T}_{\mathbf{x}}^{1}$. The contraction of $R(\mathbf{a} \wedge \mathbf{b})$ with respect to the tangent vector a gives the Ricci curvature vector,

$$
R(\mathbf{b})=\partial_{\mathbf{a}} \cdot R(\mathbf{a} \wedge \mathbf{b})=S^{2}(\mathbf{b})
$$

[11, p. 193]. Taking a second contraction with respect to $\mathbf{b}$ gives the scalar curvature $R=\partial_{\mathbf{b}} \cdot R(\mathbf{b})$. The so-called Bianchi identities take the form

$$
\begin{gathered}
R(\mathbf{a} \wedge \mathbf{b}) \cdot(\mathbf{c} \wedge \mathbf{d})=(\mathbf{a} \wedge \mathbf{b}) \cdot R(\mathbf{c} \wedge \mathbf{d}), \\
\partial_{\mathbf{a}} \wedge R(\mathbf{a} \wedge \mathbf{b})=0, \text { First Bianchi identity } \\
\partial_{\mathbf{v}} \wedge R_{\mathbb{x}}(\mathbf{a} \wedge \mathbf{b})=0 \text {, Second Bianchi identity }
\end{gathered}
$$

and can be easily established [11, p. 191].

It is well known that the Riemann curvature tensor is an intrinsic geometric quantity, independent of any extrinsic property that the manifold might inherit because of its embedding in $\mathbb{R}^{p, q}$.

\section{The Levi-Civita Coderivative}

There is another intrinsic way of deriving Riemann curvature by utilizing the Levi-Civita covariant derivative, or coderivative, defined by restricting the range and domain of the Koszul connection $\mathrm{a} \cdot \partial_{\mathrm{x}}$ to the tangent algebra $\mathcal{T}_{\mathrm{x}}$ [14, pp. 165, 503]. Formally, for any differentiable geometric valued function $F=F(\mathbf{x})$ on $\mathcal{M}$ and any $\mathbf{a} \in \mathcal{T}_{\mathbf{x}}^{1}$, we define (14)

$$
F_{\mathbf{Q}}=\mathbf{a} \cdot \nabla_{\mathbf{x}} F=P_{\mathbf{x}}\left(P_{\mathbf{x}}(F)\right)_{\mathbf{a}}=P_{\mathbf{x}}\left(\mathbf{a} \cdot \partial_{\mathbf{x}} P_{\mathbf{x}}(F(\mathbf{x}))\right) .
$$


We then naturally define the vector coderivative of $F=F(\mathbf{x})$ by

$$
\nabla F=\partial_{\mathbf{v}} F_{\mathbf{v}} .
$$

The Riemann curvature operator

$$
(\mathbf{a} \wedge \mathbf{b}) \cdot\left(\nabla_{\mathbf{x}} \wedge \nabla_{\mathbf{x}}\right)
$$

takes any tangent multivector field $A \in \mathcal{T}_{\mathrm{x}}$ into the tangent multivector field $R(\mathbf{a} \wedge \mathbf{b}) \dot{\times} A$, where

$$
R(\mathbf{a} \wedge \mathbf{b}) \dot{\times} A:=\frac{1}{2}(R(\mathbf{a} \wedge \mathbf{b}) A-A R(\mathbf{a} \wedge \mathbf{b})) .
$$

We have

$$
\begin{gathered}
(\mathbf{a} \wedge \mathbf{b}) \cdot\left(\nabla_{\mathbf{x}} \wedge \nabla_{\mathbf{x}}\right) A(\mathbf{x})=([\mathbf{b} \cdot \nabla, \mathbf{a} \cdot \nabla]-[\mathbf{b}, \mathbf{a}] \cdot \nabla) A \\
=\left(P_{\mathbf{b}} P_{\mathbf{a}}-P_{\mathbf{a}} P_{\mathbf{b}}\right)(A)=R(\mathbf{a} \wedge \mathbf{b}) \dot{\times} A,
\end{gathered}
$$

where $\mathbf{a}, \mathbf{b} \in \mathcal{T}_{\mathbf{x}}^{1}$ and $A=A(\mathbf{x})$ is any tangent multivector field with values in the tangent algebra $\mathcal{T}_{\mathbf{x}}$. For example, for $\mathbf{c} \in \mathcal{T}_{\mathbf{x}}$, we have, using (13),

$$
\begin{gathered}
R(\mathbf{a} \wedge \mathbf{b}) \cdot \mathbf{c}=\left[\partial_{\mathbf{v}} \wedge P_{\mathbf{a}} P_{\mathbf{b}}(\mathbf{c})\right] \cdot \mathbf{c} \\
=\partial_{\mathbf{v}} P_{\mathbf{a}} P_{\mathbf{b}}(\mathbf{v}) \cdot \mathbf{c}-P_{\mathbf{a}} P_{\mathbf{b}}(\mathbf{c})=\left(P_{\mathbf{b}} P_{\mathbf{a}}-P_{\mathbf{a}} P_{\mathbf{b}}\right)(\mathbf{c}) .
\end{gathered}
$$

\section{Mappings between Surfaces}

We now come to the heart of this article. Let

$$
f: \mathcal{M} \rightarrow \mathcal{M}^{\prime}, \mathbf{x}^{\prime}=f(\mathbf{x}),
$$

be a smooth mapping between two regular $k$ surfaces in $\mathbb{R}^{p, q}$. If $\mathbf{x}=\mathbf{x}\left(s^{1}, \ldots, s^{k}\right)$, then the corresponding point $\mathbf{x}^{\prime}$ is given by

$$
\mathbf{x}^{\prime}=\mathbf{x}^{\prime}\left(s^{1}, \ldots, s^{k}\right):=f\left(\mathbf{x}\left(s^{1}, \ldots, s^{k}\right)\right) .
$$

The tangent algebras $\mathcal{T}_{\mathbf{x}}$ and $\mathcal{T}_{\mathbf{x}^{\prime}}$ are related by the push forward differential defined by

$$
\mathbf{a}^{\prime}=\underline{f}(\mathbf{a})=\mathbf{a} \cdot \partial_{\mathbf{x}} f(\mathbf{x}),
$$

extended to an outermorphism by the rule

$$
\underline{f}\left(\mathbf{a}_{1} \wedge \cdots \wedge \mathbf{a}_{r}\right)=\underline{f}\left(\mathbf{a}_{1}\right) \wedge \cdots \wedge \underline{f}\left(\mathbf{a}_{r}\right) .
$$

The adjoint outermorphism is defined via the inner product to satisfy

$$
\underline{f}(\mathbf{a}) \cdot \mathbf{b}^{\prime}=\mathbf{a} \cdot \bar{f}\left(\mathbf{b}^{\prime}\right) \Leftrightarrow \bar{f}\left(\mathbf{a}^{\prime}\right)=\partial_{\mathbf{v}} \underline{f}(\mathbf{v}) \cdot \mathbf{a}^{\prime}
$$

for all $\mathbf{a}, \mathbf{b} \in \mathcal{T}_{\mathbf{x}}^{1}$ and $\mathbf{b}^{\prime} \in \mathcal{T}_{\mathbf{x}^{\prime}}^{1}$.

The chain rule

$$
\mathbf{a} \cdot \partial_{\mathbf{x}}=\underline{f}(\mathbf{a}) \cdot \partial_{\mathbf{x}^{\prime}} \Longleftrightarrow \bar{f}\left(\partial_{\mathbf{x}^{\prime}}\right)=\partial_{\mathbf{x}}
$$

relates differentiation in $\mathcal{M}$ to differentiation in $\mathcal{M}^{\prime}$.

We now wish to examine the deep relationship between intrinsic codifferentiation in $\mathcal{M}$ and intrinsic codifferentiation in $\mathcal{M}^{\prime}$. The intrinsic first coderivative of the mapping $\underline{f}$ is defined by

$$
\underline{f}_{\mathrm{a}}=\mathbf{a} \cdot \nabla \underline{f}=P^{\prime} \underline{f}_{\mathrm{a}} P,
$$

and the second coderivative by

$$
\underline{f}_{a, b}=P^{\prime}\left(\underline{f}_{\mathfrak{a}}\right)_{\mathbf{b}} P-P^{\prime} \underline{f}_{\mathbf{b} \cdot \nabla \mathbf{a}} P .
$$

Whereas the integrability condition (10) tells us that $\underline{f}_{\mathrm{a}, \mathbf{b}}-\underline{f}_{\mathbf{b}, \mathbf{a}}=0$ for ordinary differentiation, for codifferentiation we find the fundamental relationship

$$
\underline{f}_{\mathbf{a}, \mathbf{b}}-\underline{f}_{\mathbf{b}, \mathbf{a}}=\left[P_{\mathbf{b}}^{\prime}, P_{\mathbf{a}}^{\prime}\right] \underline{f}-\underline{f}\left[P_{\mathbf{b}}, P_{\mathbf{a}}\right] .
$$

Applying both sides of this relationship to the tangent vector $\mathbf{c} \in \mathcal{T}_{\mathbf{x}}^{1}$ gives

(15) $\left(\underline{f}_{\underline{a}, \mathbf{b}}-\underline{f}_{b, \mathbf{b}}\right)(\mathbf{c})=\mathbf{R}^{\prime}\left(\mathbf{a}^{\prime} \wedge \mathbf{b}^{\prime}\right) \cdot \mathbf{c}^{\prime}-\underline{\mathbf{f}}(\mathbf{R}(\mathbf{a} \wedge \mathbf{b}) \cdot \mathbf{c})$, where $\mathbf{a}^{\prime} \wedge \mathbf{b}^{\prime}=\underline{f}(\mathbf{a} \wedge \mathbf{b})$. This tells us how the curvature tensors on $\mathcal{M}$ and $\mathcal{M}^{\prime}$ are related by the coderivatives of the differential $\underline{f}$ of the mapping $f(\mathbf{x})$ between them.

\section{Conformal Mapping}

In order to justify this tour de force introduction to the methods of geometric algebra in differential geometry, we will show how deep relationships regarding conformal mappings are readily at hand. We say that $\mathbf{x}^{\prime}=f(\mathbf{x})$ is a sense-preserving conformal mapping between the surfaces $\mathcal{M}$ and $\mathcal{M}^{\prime}$ if for all $\mathbf{a}, \mathbf{b} \in \mathcal{T}_{\mathbf{x}}$ and corresponding $\mathbf{a}^{\prime}=\underline{f}(\mathbf{a})$ and $\mathbf{b}^{\prime}=\underline{f}(\mathbf{b})$ in $\mathcal{T}_{\mathbf{x}^{\prime}}$,

(16) $\underline{f}(\mathbf{a}) \cdot \underline{f}(\mathbf{b})=e^{2 \phi} \mathbf{a} \cdot \mathbf{b} \Longleftrightarrow \underline{f}(\mathbf{a})=\psi \mathbf{a} \psi^{\dagger}$, where $\phi=\phi(\mathbf{x})$ is the real-valued dilation factor, and the spinor $\psi=e^{\frac{\phi}{2}} U$ and $U U^{\dagger}=1$. The factor $U$ determines the rotation part of the conformal mapping $f(\mathbf{x})$.

Whereas we cannot give here all the explicit calculations, let us summarize the crucial results of the analysis. It turns out that for the general conformal mapping $f(\mathbf{x})$ satisfying (16) all quantities are completely determined by the dilation factor $\phi$ and its derivatives. Letting $\mathbf{w}=\partial_{\mathrm{x}} \phi$, we find that

$$
\begin{gathered}
\mathbf{a} \cdot \mathbf{w}=\frac{1}{k} \partial_{\mathbf{v}^{\prime}} \cdot{\underline{\mathbf{v}^{\prime}}}_{\mathbf{v}^{\prime}}(\mathbf{a}), \quad U_{\grave{a}}=U \mathbf{a} \wedge \mathbf{w}, \\
\psi_{\mathfrak{a}}=\frac{1}{2} \psi(\mathbf{a} \cdot \mathbf{w}+\mathbf{a} \wedge \mathbf{w})=\frac{1}{2} \psi \mathbf{a w}, \\
\underline{f}_{\mathbf{b}}(\mathbf{a})=\frac{1}{2} \underline{f}(\mathbf{a} \mathbf{w} \mathbf{b}+\mathbf{b} \mathbf{w} \mathbf{a}),
\end{gathered}
$$

and

$$
\left(\underline{f}_{\mathbf{a}, \mathbf{b}}-\underline{f}_{b, \mathbf{a}}\right)(\mathbf{c})=\underline{f}(\Omega \cdot \mathbf{c})
$$

where

$$
\Omega=(\mathbf{a} \wedge \mathbf{b} \wedge \mathbf{w}) \mathbf{w}+(\mathbf{a} \wedge \mathbf{b}) \cdot \nabla \mathbf{w} .
$$

Using (15) and taking the outer product of both sides of (17) with $\underline{f}\left(\partial_{\mathbf{c}}\right)=e^{2 \phi} \partial_{\mathbf{c}^{\prime}}$ gives the important relationship

We call

$$
\underline{f}(\Omega)=e^{2 \phi} R^{\prime}\left(\mathbf{a}^{\prime} \wedge \mathbf{b}^{\prime}\right)-\underline{f}(R(\mathbf{a} \wedge \mathbf{b})) .
$$

$$
\mathcal{W}_{4}(\mathbf{a} \wedge \mathbf{b})=R(\mathbf{a} \wedge \mathbf{b}) \wedge(\mathbf{a} \wedge \mathbf{b})
$$

the Weyl 4-vector because it is closely related to the classical conformal Weyl tensor, which is important in Einstein's general relativity [17], [25]. 
Notice that the Weyl 4-vector vanishes identically if the dimension of the $k$-manifold is less than 4. Taking the outer product of both sides of (18) with $\underline{f}(\mathbf{a} \wedge \mathbf{b})=\mathbf{a}^{\prime} \wedge \mathbf{b}^{\prime}$, and noting that $\Omega \wedge \mathbf{a} \wedge \mathbf{b}=0$, gives the relationship

$$
\underline{f}\left(\mathcal{W}_{4}(\mathbf{a} \wedge \mathbf{b})\right)=e^{2 \phi} \mathcal{W}_{4}^{\prime}\left(\mathbf{a}^{\prime} \wedge \mathbf{b}^{\prime}\right) \text {. }
$$

The classical conformal Weyl tensor can be identified with

$$
\begin{aligned}
\mathcal{W}_{C}(\mathbf{a} \wedge \mathbf{b})=\frac{1}{(k-1)(k-2)}\left(\partial_{\mathbf{v}} \wedge \partial_{\mathbf{u}}\right) \cdot \mathcal{W}_{4}(\mathbf{u} \wedge \mathbf{v}) \\
=R(\mathbf{a} \wedge \mathbf{b})-\frac{1}{k-2}[R(\mathbf{a}) \wedge \mathbf{b}+\mathbf{a} \wedge R(\mathbf{b})] \\
+\frac{R \mathbf{a} \wedge \mathbf{b}}{(k-1)(k-2)} .
\end{aligned}
$$

Taking the contraction of both sides of (19) with $\partial_{\mathbf{b}} \wedge \partial_{\mathbf{a}}$ gives the relation

$$
\underline{f}\left(\mathcal{W}_{C}(\mathbf{a} \wedge \mathbf{b})\right)=e^{2 \phi} \mathcal{W}_{C}^{\prime}\left(\mathbf{a}^{\prime} \wedge \mathbf{b}^{\prime}\right)
$$

which is equivalent to

$$
\underline{f}\left(\mathcal{W}_{C}(\mathbf{a} \wedge \mathbf{b}) \cdot \mathbf{c}\right)=\mathcal{W}_{C}^{\prime}\left(\mathbf{a}^{\prime} \wedge \mathbf{b}^{\prime}\right) \cdot \mathbf{c}^{\prime}
$$

where $\mathbf{c}^{\prime}=\underline{f}(\mathbf{c})$.

When we make the assumption that our conformal mapping $\mathbf{x}^{\prime}=f(\mathbf{x})$ is between the flat space $\mathcal{M}=\mathbb{R}_{p, q}=\mathcal{M}^{\prime}$ and itself, for which the curvature bivectors $R(\mathbf{a} \wedge \mathbf{b})$ and $R^{\prime}\left(\mathbf{a}^{\prime} \wedge \mathbf{b}^{\prime}\right)$ vanish, (18) simplifies to the simple equation that $\Omega \equiv 0$. Taking the contraction of this equation with respect to the bivector variable $\mathbf{B}=\mathbf{a} \wedge \mathbf{b}$ gives the relationship

$$
\nabla \cdot \mathbf{w}=-\frac{k-2}{2} \mathbf{w}^{2}
$$

for all values of $k=n>2$. Calculating $\partial_{\mathbf{b}} \cdot \Omega=0$ and eliminating $\nabla \cdot \mathbf{w}$ from this equation leads to the surprisingly simple differential equation

$$
\mathbf{w}_{\mathbf{a}}=\mathbf{a} \cdot \nabla \mathbf{w}=\frac{1}{2} \mathbf{w a w} .
$$

The equation (20) specifies the extra condition that $\mathbf{w}=\nabla \phi$ must satisfy in order for $f(\mathbf{x})$ to be a nondegenerate conformal mapping in the pseudoeuclidean space $\mathbb{R}^{p, q}$ onto itself, where $n=p+q>2$.

Trivial solutions of (20) satisfying (16) consist of (i) $\nabla \phi=0$ so that $\psi$ is a constant dilation factor and (ii) $\psi=U$ where $U$ is a constant rotation in the plane of some constant bivector $\mathbf{B}$.

Let $\mathbf{c}$ be a constant nonnull vector in $\mathbb{R}^{p, q}$. A nontrivial solution to (20) is

$$
f(x)=\psi \mathbf{x}=\mathbf{x}(1-\mathbf{c x})^{-1}=\frac{1}{2} \mathbf{c}^{-1} \mathbf{w} \mathbf{x}
$$

where $\mathbf{w}=\nabla \phi=2(1-\mathbf{c x})^{-1} \mathbf{c}$ and

$$
e^{-\phi}=(1-\mathbf{x c})(1-\mathbf{c x})=4 \mathbf{c}^{2} \mathbf{W}^{2} \text {. }
$$

Equivalently, we can write (21) in the form

$$
f(\mathbf{x})=\frac{\mathbf{x}-\mathbf{x}^{2} \mathbf{c}}{1-2 \mathbf{c} \cdot \mathbf{x}+\mathbf{c}^{2} \mathbf{x}^{2}} .
$$

The mapping $f(\mathbf{x})$ is called a transversion in the identity component of the conformal or Möbius group of $\mathbb{R}^{p, q}$. In addition to transversions, the sense-preserving conformal group is generated by rotations, translations, and dilations. A related, more detailed, derivation of the sense-preserving conformal group can be found in [11, pp. 210-19].

\section{Möbius Transformations and Vahlen Matrices}

We must take a step back to see the whole picture of the structure of conformal transformations in $\mathbb{R}^{p, q}$. Following [15, pp. 248-51], by the Vahlen matrix $[f]$ of a conformal transformation of the form $f(\mathbf{x})=(a \mathbf{x}+b)(c \mathbf{x}+d)^{-1}$ where $a, b, c, d \in \mathbb{G}_{p, q}$, we mean

$$
[f]=\left(\begin{array}{ll}
a & b \\
c & d
\end{array}\right) .
$$

The Vahlen matrix $[f]$ of the identity transformation $f(\mathbf{x})=\mathbf{x}$ is easily seen to be the identity $\operatorname{matrix}[f]=\left(\begin{array}{ll}1 & 0 \\ 0 & 1\end{array}\right)$.

The Vahlen matrices of conformal transformations greatly simplify the study of the conformal group, because the group action of composition is reduced to the matrix product of the corresponding Vahlen matrices. If $f(\mathbf{x})$ and $g(\mathbf{x})$ are conformal transformations, then $h(\mathbf{x})=g(f(\mathbf{x}))$ is also a conformal transformation of $\mathbb{R}^{p, q}$ and their Vahlen matrices satisfy the rule

$$
[h]=[g \circ f]=[g][f] .
$$

By the pseudodeterminant of a Vahlen matrix, we mean

$$
\operatorname{pdet}\left(\begin{array}{ll}
a & b \\
c & d
\end{array}\right)=a d^{\dagger}-b c^{\dagger} \text {. }
$$

A conformal transformation $f$ is sense-preserving (rotation) if $p \operatorname{det}[f]>0$ and sense reversing (reflection) if $p \operatorname{det}[f]<0$. By the normalized identity component of the conformal group, we mean all conformal transformations $f$ with the property that $p \operatorname{det}[f]=1$.

We can now talk about the conformal Möbius transformations shown in Figures 6-10. Figures 6,7 , and 8 all have Vahlen matrices of the form $\left(\begin{array}{cc}1 & 0 \\ -\mathbf{c} & 1\end{array}\right)$ with the pseudodeterminant

$$
\operatorname{pdet}\left(\begin{array}{cc}
1 & 0 \\
-\mathbf{c} & 1
\end{array}\right)=1 \text {. }
$$

This means that these transformations are sensepreserving conformal transformations of $\mathbb{R}^{3}$ onto itself which are continuously connected to the identity component represented by the identity $2 \times 2$ matrix. 


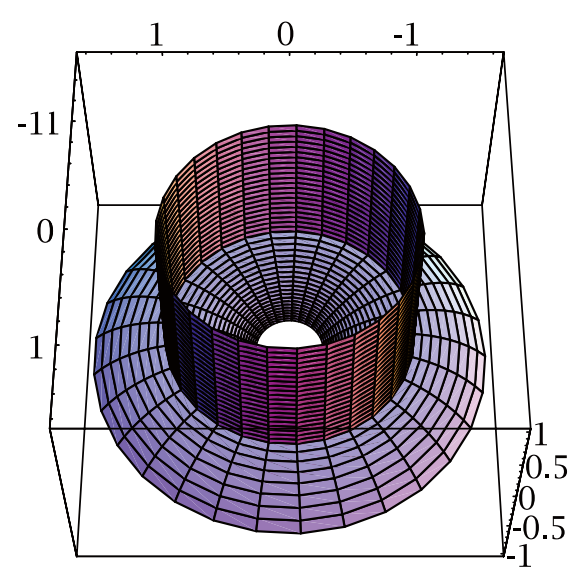

Figure 6. The transversion $f(\mathbf{x})=\mathbf{x}(1-\mathbf{c x})^{-1}$, with $\mathrm{c}=(0,0,3 / 4)$, maps the cylinder in $\mathbb{R}^{3}$ conformally onto the figure shown.

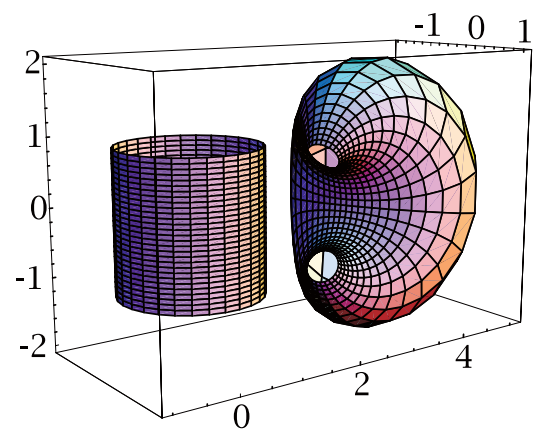

Figure 7. The transversion $f(\mathbf{x})=\mathbf{x}(1-\mathbf{c x})^{-1}$ with $\mathbf{c}=(-1 / 4,1 / 2,0)$ maps the cylinder in $\mathbb{R}^{3}$ conformally onto the figure shown. The image is translated by 3 units on the $y$-axis for clarity.

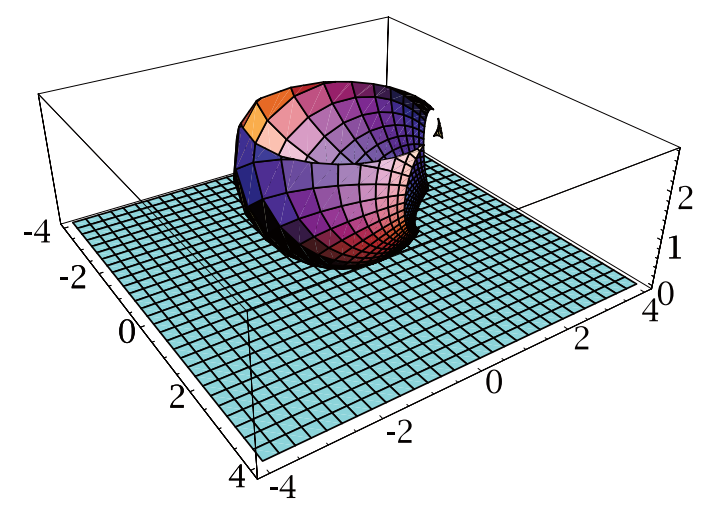

Figure 8. The transversion $f(\mathbf{x})=\mathbf{x}(1-\mathbf{c x})^{-1}$ with $\mathrm{c}=(-1 / 4,-1 / 4,-1 / 4)$ maps the $x y$-plane conformally onto the unit sphere in $\mathbb{R}^{3}$ above the plane as shown.

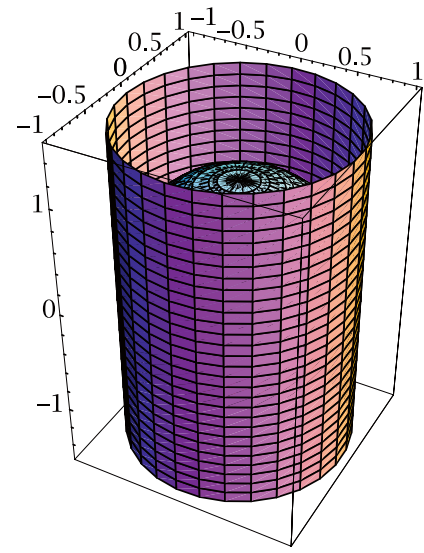

Figure 9. The stereographic projection $f(\mathbf{x})=(\mathbf{c x}+1)(\mathbf{x}-\mathbf{c})^{-1}$ in $\mathbb{R}^{3}$, with $\mathbf{c}=(0,0,1)$, wraps the unit cylinder conformally around the unit sphere as shown. For a cylinder 4 times as long as the radius of the sphere, the sphere is covered twice.

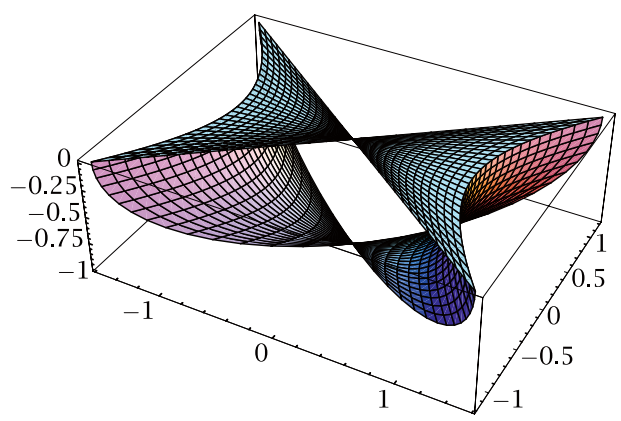

Figure 10. The hyperbolic stereographic projection $f(\mathbf{x})=(\mathbf{x}-\mathbf{c})(1+\mathbf{c x})^{-1}$ with $c=(0,0,1)$ maps the hyperbolic $x y$-plane conformally onto the unit hyperboloid. The metric $g$ has the Lorentzian signature $(+,-,+)$.

Figures 9 and 10 have Vahlen matrices of the respective forms

$$
\left(\begin{array}{cc}
\mathbf{c} & 1 \\
1 & -\mathbf{c}
\end{array}\right) \text { and }\left(\begin{array}{cc}
1 & -\mathbf{c} \\
\mathbf{c} & 1
\end{array}\right)
$$

with pseudodeterminants -2 and 2, respectively. These global conformal transformations on $\mathbb{R}^{3}$ and $\mathbb{R}^{2,1}$ are the extensions of the standard stereographic projection from the Euclidean plane in $\mathbb{R}^{2}$ to the unit sphere in $\mathbb{R}^{3}$ in the case of Figure 9 , and from the hyperbolic plane in $\mathbb{R}^{1,1}$ to the unit hyperboloid in $\mathbb{R}^{2,1}$ in the case of Figure 10 . Whereas Figure 10 is continuously connected to the identity component as $\mathbf{c} \rightarrow 0$, this is not the case for Figure 9.

We wish to call one peculiarity to the attention of the reader. The standard stereographic transformation from the Euclidian plane in $\mathbb{R}^{2}$ to the unit sphere in $\mathbb{R}^{3}$, with the north pole at 
the unit vector $\mathbf{e}_{3}$ on the $z$-axis, can be represented either by $f(\mathbf{x})=\left(\mathbf{e}_{3} \mathbf{x}+1\right)\left(\mathbf{x}-\mathbf{e}_{3}\right)^{-1}$ or by $g(\mathbf{x})=\left(\mathbf{x}-\mathbf{e}_{3}\right)\left(\mathbf{e}_{3} \mathbf{x}+1\right)^{-1}$. Both of these transformations are identical when restricted to the $x y$-plane, but are globally distinct on $\mathbb{R}^{3}$. One of these conformal transformations is sense preserving and continuously connected to the identity, while the other one is not. How is this possible?

For the reader who wants to experiment with these ideas but is not so familiar with geometric algebra, I highly recommend the Clifford algebra calculator software [16], which can be downloaded. The reader may find it interesting to compare our methods and results to those found in $[8, \mathrm{pp}$. 106-118].

At the website http: //www.garretstar.com/ algebra can be found supplementary material, including the Links referred to in this article and to other related websites, a discussion of the horosphere explaining the deep relationship between the Vahlen matrices of elements in $G_{p, q}$ to the orthogonal group of $R^{p+1, q+1}$, and figures further illustrating the richness of conformal mappings. At present, the username: garretams and password: garretams are required to view this material. Also included is a list of additional references to the literature to give the reader a better idea of the many different applications that Clifford algebras have found in mathematics, theoretical physics, and in the computer science and engineering communities.

\section{Acknowledgments}

The author wants to thank the reviewers of this article for their helpful comments, which led to improvements, and to the Universidad de Las Américas-Puebla and to CONACYT SNI Exp. 14587 for their many years of support.

\section{References}

[1] R. ABlamowicz and G. SobczyK, Lectures on Clifford (Geometric) Algebras and Applications, Birkhäuser, Boston, 2004.

[2] H. Blaine LAwSON and M. L. Michelson, Spin Geometry, Princeton University Press, Princeton, 1989.

[3] F. Brackx, R. Delanghe, F. Sommen, Clifford Analysis, Pitman Publishers, Boston-London-Melbourne, 1982.

[4] C. J. S. ClaRKE, On the global isometric embedding of pseudo-Riemannian manifolds, Proc. Roy. Soc. A 314, 417-428 (1970).

[5] T. DANTZIG, NUMBER: The Language of Science, Fourth Edition, Free Press, 1967.

[6] T. F. HAVEL, Geometric Algebra: Parallel Processing for the Mind, Nuclear Engineering, 2002. See Links 1.

[7] J. GILBERT and M. Murray, Clifford Algebras and Dirac Operators in Harmonic Analysis, Cambridge Studies in Advanced Mathematics, Cambridge University Press, Cambridge, 1991 (Online publication date: November 2009).
[8] S. I. Goldberg, Curvature and Homology, Dover Publications, New York, 1982.

[9] D. HestenES, New Foundations for Classical Mechanics, 2nd Ed., Kluwer, 1999.

[10] _ Grassmann's Legacy, in Grassmann Bicentennial Conference (1809-1877), September 16-19, 2009, Potsdam-Szczecin (DE, PL). See Links 2.

[11] D. Hestenes and G. SoBczyK, Clifford Algebra to Geometric Calculus: A Unified Language for Mathematics and Physics, 3rd edition, Kluwer, 1992.

[12] N. J. Hicks, Notes on Differential Geometry, Van Nostrand, Princeton, NJ, 1965.

[13] D. Husemöller, Fibre Bundles, McGraw-Hill, New York, 1966.

[14] J. M. LEE, Manifolds and Differential Geometry, Graduate Studies in Mathematics, Vol. 107, American Mathematical Society, Providence, RI, 2009.

[15] P. Lounesto, Clifford Algebras and Spinors, 2nd edition, Cambridge University Press, Cambridge, 2001. [16] _ CLICAL Algebra Calculator and Software, Helsinki University, 1987. See Links 3.

[17] C. W. Misner, K. S. THORNE and J. A. WHEELER, Gravitation, Freeman and Company, San Francisco, CA, 1973.

[18] P. NAHIN, An Imaginary Tale: The Story of the Square Root of Minus One, Princeton University Press, Princeton, NJ, 1998.

[19] J. NASH, $C^{1}$ isometric imbeddings, Annals of Mathematics (2), 60(3):383-396, 1954.

[20] _ The imbedding problem for Riemannian manifolds, Annals of Mathematics 2, 63(1):20-63, 1956. [21] I. R. PORTEOUS, Clifford Algebras and the Classical Groups, Cambridge University Press, Cambridge, 1995. [22] G. Soвczyк, Hyperbolic number plane, The College Mathematics Journal, 26(4):269-280, September 1995.

[23] _ , Geometric matrix algebra, Linear Algebra and its Applications, 429:1163-1173, 2008.

[24] _ New Foundations in Mathematics: The Geometric Concept of Number, San Luis Tehuiloyocan, Mexico, 2010. See Links 4.

[25] _ Plebanski classification of the tensor of matter, Acta Physica Polonica, B11(6), 1981.

[26] _ Mappings of Surfaces in Euclidean Space Using Geometric Algebra, Ph.D dissertation, Arizona State University, 1971. See Links 5.

[27] J. PozO and G. SoвCZYK, Geometric algebra in linear algebra and geometry, Acta Applicandae Mathematicae, 71: 207-244, 2002.

[28] M. SpIVAK, Calculus on Manifolds, W. A. Benjamin, Inc., New York, 1965.

[29] D. J. STRUIK, A Concise History of Mathematics, Dover, 1967.

[30] N. Verma, Towards an Algorithmic Realization of Nash's Embedding Theorem, CSE, UC San Diego. See Links 6.

[31] E. WitTen, Magic, mystery, and matrix, Notices Amer. Math. Soc., 45(9):1124-1129, 1998.

[32] H. Whitney, Differentiable manifolds, Annals of Mathematics (2), 37:645-680, 1936. 